\title{
Laser spectroscopy studies on nobelium
}

\author{
Michael Block ${ }^{1,2,3, \star}$ \\ 1 GSI Helmholtzzentrum für Schwerionenforschung, Planckstrasse 1, 64291 Darmstadt, Germany \\ ${ }^{2}$ Helmholtz Institut Mainz, Staudingerweg 18, 55128 Mainz, Germany \\ ${ }^{3}$ Johannes Gutenberg-Universität Mainz, 55099 Mainz, Germany
}

\begin{abstract}
Laser spectroscopy of the heaviest elements provides high-precision data on their atomic and nuclear properties. For example, atomic level energies and ionization potentials allow us to probe the influence of relativistic effects on their atomic structure and to benchmark state-of-the-art atomic structure calculations. In addition, it offers an alternative route to determine nuclear properties like spins, magnetic moments and quadrupole moments in a nuclear model-independent way. Recently, a sensitive method based on resonant laser ionization has been applied to nobelium isotopes around $N=152$ at GSI Darmstadt. In pioneering experiments, several atomic states have been identified extending the reach of laser spectroscopy beyond fermium. In this contribution, the main achievements and future perspectives are briefly summarized.
\end{abstract}

\section{Introduction}

The atomic and chemical properties of the heaviest elements $(Z \gtrsim 100)$ are affected by strong relativistic effects and quantum electrodynamics [1-3]. Relativistic effects increase approximately with the square of the atomic number and are responsible for the distinct color of gold and for the liquid state of mercury at room temperature. For example, these effects stabilize $\mathrm{s}$ and $\mathrm{p}_{1 / 2}$ orbitals, whereas $\mathrm{p}_{3 / 2}$ and $\mathrm{d}$ orbitals are destabilized in energy. This leads to changes of the atomic ground state configurations compared to the trend observed in the elements we find in nature. In lawrencium $(Z=103)$, for example, a $7 p_{1 / 2}$ ground state is predicted instead of a $6 \mathrm{~d}$ state [4]. At some point, a deviation from the regular pattern that governs the ordering of elements in the periodic table is expected. Such deviations were predicted for the superheavy elements copernicium $(Z=112)$ and flerovium $(Z=114)$ in the mid 1970s [5]. According to Pitzer these elements should already show properties of noble gases even though the next nobel gas in a regular periodic table would be oganesson $(Z=118)$.

Experimentally, atomic and chemical properties of the heaviest elements have been mainly studied by chemistry techniques either in gas phase or in liquid phase [68]. Copernicium was actually found to be a rather regular member of group twelve [9], while the properties of flerovium are still a matter of ongoing research $[10,11]$.

An alternative approach to study atomic properties of heavy elements is through laser spectroscopy. Wellestablished techniques provide high-precision data on atomic properties such as atomic level energies, lifetimes and ionization potentials that allow us to benchmark theo-

\footnotetext{
^e-mail:m.block@gsi.de
}

retical predictions and to probe the influence of relativistic effects. Also, nuclear properties are reflected in the atomic spectrum. This enables studies of the evolution of nuclear structure features in a complementary way to traditional nuclear spectroscopy. For example, hyperfine spectroscopy give access to the nuclear spin and to nuclear moments. The shift of an atomic transition in different isotopes allows determining changes in mean square charge radii of nuclei and hence, the nuclear size and deformation in a nuclear model-independent way. However, accurate calculations of the hyperfine parameters $A$ and $B$ or the field shift factor $F$ are required inputs from atomic theory.

\section{Resonant ionization laser spectroscopy of heavy nuclides}

The method of choice for optical spectroscopy of rare isotopes is resonant laser ionization spectroscopy (RIS). This technique features high sensitivity, in particular if the laser-created ions are detected by their characteristic radioactive decay. The method can even be applied to atoms where no experimental information on atomic transitions exists, which had been illustrated in fermium [12] and, more recently, in astatine [13], for example.

For more than a decade, fermium $(Z=100)$ was the heaviest element that had been studied by laser spectroscopy [12]. A tiny quantity of $46 \mathrm{pg}$ of the long-lived isotope ${ }^{255} \mathrm{Fm}\left(T_{1 / 2} \approx 20 \mathrm{~h}\right)$ was prepared in the highflux reactor at Oak Ridge National laboratory. This enabled offline experiments in which several atomic transitions were identified. Good agreement with predictions by multi-configuration Dirac-Fock (MCDF) calculations [14] was observed. In addition, broadband spectroscopy of the complex hyperfine spectrum of ${ }^{255} \mathrm{Fm}$ with a nuclear spin 
of $I=7 / 2$ was feasible and the hyperfine factors $A$ and $B$ were obtained from a fit to the data [15].

The heavier members of the actinide series beyond fermium have to be studied online. However, these elements cannot be produced at isotope separator online (ISOL) facilities, but in fusion-evaporation reactions. This is experimentally challenging due to low production rates on the order of at most few particles per second. In addition, the radionuclides have to be slowed down from tens of $\mathrm{MeV}$ to rest and they have to be neutralized to perform RIS. This is nowadays accomplished using gas cells filled with an inert buffer gas like argon.
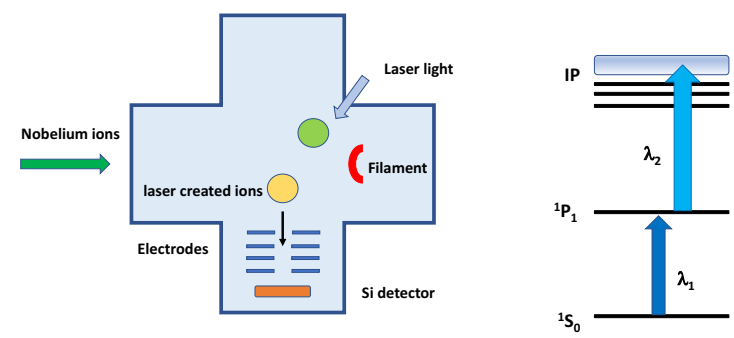

Figure 1. Left: Schematic view of the buffer gas cell for laser spectroscopy. Right: Simplified laser excitation scheme.

A dedicated RIS version, the so-called radiation detected resonance ionization spectroscopy method (RADRIS), has been developed for nobelium laser spectroscopy at GSI Darmstadt $[16,17]$. The setup is schematically shown in 1 . In the RADRIS method, nobelium ions are separated from the primary beam by the separator for heavy ion reaction products (SHIP [18, 19]) and slowed down in 100 mbar ultra-pure argon gas. The fraction of the stopped ions that remains in a charged state is accumulated on a tantalum filament. Neutral nobelium atoms are then evaporated by heating this filament. The atoms are ionized in a two-step laser excitation scheme with pulsed lasers. For the initial level search the first step is provided by a tunable dye laser that is scanned across the spectral region in which atomic states were predicted. The second excitation step into the continuum is provided by a fixedfrequency excimer laser (at $351 \mathrm{~nm}$ ) featuring high power to compensate the lower cross section of a non-resonant excitation. The laser-created ions are detected by their characteristic $\alpha$ decay registered by a silicon detector to which the ions are transported by electric fields. Details on the method and the employed setup have been described elsewhere [20].

\section{Scientific questions addressed by laser spectroscopy}

Detailed studies of the nuclear structure and its evolution in regions of enhanced shell stabilization are of great importance for a better understanding of the heaviest elements. Their very existence is intimately linked to nuclear shell effects. Ultimately, such investigations will shed light on the nature of the underlying strong interaction at one of the extremes of the nuclear chart. However, the nuclides at the predicted spherical closed shells with $N=184$ and $Z=114,120$, or 126 [21] are experimentally still inaccessible at present. The most neutron-rich nuclide known to date contains only 177 neutrons [22]. In addition, detailed studies of the elements with $Z \geq 110$ are hampered by limited statistics due to low cross sections on a level of 1-10 picobarn.

Lighter nuclides in the region around $Z=100, N=$ 152 can be produced with higher rates that allow Penning trap-mass spectrometry [23], laser spectroscopy [24] and $\alpha-\gamma$ spectroscopy [25]. Indeed, some of the single-particle orbitals that are responsible for the spherical shell gap at $Z=114$ appear at low excitation energy in deformed nuclei around $Z \approx 100, N=152$. Thus, by fixing the position of these orbitals at a given deformation allows discriminating models predicting the spherical gap. This region also forms the backbone for spin assignments in heavier nuclides in decay spectroscopy experiments based on systematics along isotones.

A complementary determination of nuclear spins by laser spectroscopy is desirable. In addition, measurements of dipole and quadrupole moments allow us to determine single-particle configurations in specific nuclei. This would, for example, provide an alternative to determine the configuration of the long-lived $K=8^{-}$isomer in ${ }^{254}$ No for which different interpretations have been put forward based on decay spectroscopy [26-30]. The magnetic moment that could be measured by laser spectroscopy could answer the questions whether this isomer is based on a quasi-neutron or quasi-proton configuration that can both lead to $K=8^{-}$states at similar energy.

Nobelium atoms have a favorable atomic structure for laser spectroscopy: the $5 \mathrm{f}$ shell is filled by 14 electrons and the two valence electrons in the $7 \mathrm{~s}$ orbital result in a ground-state configuration $[\mathrm{Rn}] 5 \mathrm{f}^{14} 7 \mathrm{~s}^{2}{ }^{1} \mathrm{~S}_{0}$. This facilitates accurate calculations of atomic properties. Modern calculations of the electronic structure in the heaviest elements require the explicit treatment of relativistic and quantum electrodynamics effects. In addition, electron correlations have to considered. This is accomplished employing many-body approaches like multi-configuration Dirac-Fock or relativistic coupled cluster methods [1$3,14]$. They can reach high accuracy with a typical precision of meV for level energies. The accuracy of such calculations is often estimated by a comparison of calculated properties to experimental data in the lanthanide region since the available data for the heaviest elements are limited. According to theoretical predictions nobelium features a strong ground-state transition ${ }^{1} \mathrm{~S}_{0^{-}}{ }^{1} \mathrm{P}_{1}$ around $30,000 \mathrm{~cm}^{-1}[14,31,32]$.

\section{Status and future perspectives}

A practical advantage for studies of nobelium isotopes around $N=152$ is their rather high yield: ${ }^{254}$ No can be produced in the reaction ${ }^{208} \mathrm{~Pb}\left({ }^{48} \mathrm{Ca}, 2 \mathrm{n}\right)$ with a cross section of about $2 \mu \mathrm{b}$ corresponding to a yield of few particles per second at an accelerator facility like GSI Darmstadt. 
Applying the RADRIS method to the nobelium isotope ${ }^{254} \mathrm{No}$, the strong ground state transition ${ }^{1} \mathrm{~S}_{0^{-}}{ }^{1} \mathrm{P}_{1}$ has been searched for in a tedious effort $[16,17]$. Such experiments depend crucially on the guidance by theoretical predictions. Different atomic transitions in the nobelium atom have been predicted prior to the GSI experiment $[14,31,32]$. The typical uncertainties and the scattering of the predictions resulted in scan range on the order of $\geq 2,000 \mathrm{~cm}^{-1}$ that had to be covered. For a typical line width of in-gas cell spectroscopy of about $0.5 \mathrm{~cm}^{-1}$ [16] this corresponds to a few thousand frequency steps for the initial search. In the case of ${ }^{254}$ No the cycle time for one such step sums up to about five minutes equivalent to a minimum search time of more than 200 hours to cover the range of the predicted atomic transitions.

Nonetheless, the ${ }^{1} \mathrm{~S}_{0^{-}}{ }^{1} \mathrm{P}_{1}$ transition as well as several Rydberg states in the nobelium isotope ${ }^{254}$ No have been identified for the first time recently [33]. An overall efficiency about six percent was achieved using a two-step laser excitation that could be boosted for a limited time by conditioning the filament. Based on the observed Rydberg series in ${ }^{254}$ No the first ionization potential could already be derived with rather high precision. However, quenching collisions led to the population of a metastable state, most likely the ${ }^{3} \mathrm{D}_{1}$ state that is close to the ${ }^{1} \mathrm{P}_{1}$ state that was excited by the first laser pulse. This behavior was explained by a rate equation model that describes the experimental data well and allows determining the energy of the ${ }^{3} \mathrm{D}_{1}$ state indirectly [33, 34]. However, Rydberg states could be excited by the second laser from either of the two states resulting in different series. In 2016, different Rydberg series were unambiguously identified, including a series originating form the ${ }^{1} \mathrm{P}_{1}$ state from which the first ionization potential of nobelium could be determined with high precision. The data analysis is close to completion and the results will be published shortly. All in all, the results for atomic properties of nobelium showed good agreement with theoretical predictions [14, 31, 32].

The measurements were extended to the nobelium isotopes ${ }^{252,253}$ No. The lowest yield was available for ${ }^{252}$ No with a cross section of about 400 nanobarn in the reaction ${ }^{208} \mathrm{~Pb}\left({ }^{48} \mathrm{Ca}, 2 \mathrm{n}\right){ }^{252}$ No. Decay losses on the filament reduced efficiency to about three precent for this shorterlived nobelium isotope [33]. The isotope shift of the ${ }^{1} \mathrm{~S}_{0^{-}}$ ${ }^{1} \mathrm{P}_{1}$ transition between ${ }^{252,253,254}$ No was measured and the hyperfine splitting in ${ }^{253}$ No was observed. The data analysis is ongoing and the results will be subject of forthcoming publications. The experiments will provide the change of the nuclear charge radius between the isotopes ${ }^{252,254} \mathrm{No}$ as well as the magnetic moment and the ground-state spin assignment in ${ }^{253}$ No.

Resonant laser ionization spectroscopy in a gas cell is a powerful method to identify atomic transitions in rare isotopes where little is known experimentally. However, the line width that can be achieved with in-gas cell spectroscopy is limited by pressure and Doppler broadening to typically about $5 \mathrm{GHz}$. In some cases higher resolution spectroscopy is required, for example to resolve individual hyperfine components for the determination of the magnetic moment. A higher resolution was recently demon- strated with a new technique, the so-called gas-jet laser spectroscopy [35]. The method was recently developed at $\mathrm{KU}$ Leuven and was applied to actinium isotopes where a gain in resolution by almost one order of magnitude compared to in-gas cell spectroscopy without sacrificing the high efficiency.

Future efforts will be dedicated to extend the reach of laser spectroscopy even further towards heavier elements. For a first foray into an unexplored area, a broadband level search with high-power lasers in a gas cell, for example with the RADRIS technique, will be favorable. Once suitable transitions have been identified, high-resolution spectroscopy can be performed by in-gas jet spectroscopy. In any case the steeply dropping cross section for the production of heavier elements are challenging. In this respect, laser spectroscopy will profit from new powerful stablebeam accelerators. Such machines that are anticipated to deliver 10-100 times higher primary beam intensities are presently planned or under construction in several facilities worldwide [36-38].

For the first exploration of the element lawrencium, the RADRIS method can be adapted in a straightforward manner. A key question in $\mathrm{Lr}$ that can be answered by laser spectroscopy concerns the atomic ground-state configuration as discussed above. Theoretical models predict a $7 p_{1 / 2}$ ground state, but the $6 d$ state is rather close in energy [4]. Recent experiments performed in Tokai, Japan used a surface ionization technique to determine the first ionization potential of $\operatorname{Lr}$ [39]. Their result agrees with the theoretical prediction well and confirms that $\mathrm{Lr}$ terminates the actinide series. However, the uncertainty of the employed method does not allow distinguishing between the two different ground state configurations unambiguously. Thus, an experimental verification by laser spectroscopy is still of interest.

\section{Acknowledgements}

The experiments on nobelium discussed in this contribution were performed by the RADRIS collaboration comprising scientists from GSI Darmstadt, Helmholtz Institute Mainz, Mainz University, Technical University Darmstadt, KU Leuven, University of Liverpool, and TRIUMF Vancouver. Their contributions are gratefully acknowledged.

\section{References}

[1] P. Schwerdtfeger, L.F. Pašteka, A. Punnett, P.O. Bowman, Nuclear Physics A 944, 551 (2015)

[2] E. Eliav, S. Fritzsche, U. Kaldor, Nucl. Phys. A944, 518 (2015)

[3] V. Pershina, Nuclear Physics A 944, 578 (2015)

[4] A. Borschevsky, E. Eliav, M. Vilkas, Y. Ishikawa, U. Kaldor, Eur. Phys. J. D 45, 115 (2007)

[5] K.S. Pitzer, The Journal of Chemical Physics 63, 1032 (1975)

[6] M. Schädel, Phil. Trans. R. Soc. A 373, 20140191 (2015) 
[7] A. Türler, R. Eichler, A. Yakushev, Nuclear Physics A 944, 640 (2015)

[8] Y. Nagame, J.V. Kratz, M. Schädel, Nucl. Phys. A944, 614 (2015)

[9] R. Eichler, N. Aksenov, A. Belozerov, G. Bozhikov, V. Chepigin, S. Dmitriev, R. Dressler, H. Gäggeler, V. Gorshkov, F. Haenssler et al., Nature 447, 72 (2007)

[10] R. Eichler, N. Aksenov, Y.V. Albin, A. Belozerov, G. Bozhikov, V. Chepigin, S. Dmitriev, R. Dressler, H. Gäggeler, V. Gorshkov et al., Radiochimica Acta International journal for chemical aspects of nuclear science and technology 98, 133 (2010)

[11] A. Yakushev, J.M. Gates, A. Türler, M. Schädel, C.E. Düllmann, D. Ackermann, L.L. Andersson, M. Block, W. Brüchle, J. Dvorak et al., Inorganic chemistryü 53, 1624 (2014)

[12] M. Sewtz, H. Backe, A. Dretzke, G. Kube, W. Lauth, P. Schwamb, K. Eberhardt, C. Grüning, P. Thörle, N. Trautmann et al., Phys. Rev. Lett 90, 163002 (2003)

[13] S. Rothe, V.N. Fedosseev, T. Kron, B.A. Marsh, R.E. Rossel, K.D.A. Wendt, Nucl. Instrum. Meth. B317, 561 (2013)

[14] S. Fritzsche, C. Dong, F. Koike, A. Uvarov, Eur. Phys. J. D 45, 107 (2007)

[15] H. Backe, A. Dretzke, R. Haire, P. Kunz, W. Lauth, M. Sewtz, N. Trautmann et al., Hyperfine interactions 162, 3 (2005)

[16] H. Backe, P. Kunz, W. Lauth, A. Dretzke, R. Horn, T. Kolb, M. Laatiaoui, M. Sewtz, D. Ackermann, M. Block et al., Eur. Phys. J. D 45, 99 (2007)

[17] M. Laatiaoui, H. Backe, M. Block, P. Chhetri, F. Lautenschläger, W. Lauth, T. Walther, Hyperfine Interact. 227, 69 (2014)

[18] G. Münzenberg, W. Faust, S. Hofmann, P. Armbruster, K. Güttner, H. Ewald, Nucl. Instrum. and Meth. 161, 65 (1979)

[19] S. Hofmann, G. Münzenberg, Reviews of Modern Physics 72, 733 (2000)

[20] F. Lautenschläger, P. Chhetri, D. Ackermann, H. Backe, M. Block, B. Cheal, A. Clark, C. Droese, R. Ferrer, F. Giacoppo et al., Nuclear Instruments and Methods in Physics Research Section B: Beam Interactions with Materials and Atoms 383, 115 (2016)

[21] A. Sobiczewski, K. Pomorski, Progress in Particle and Nuclear Physics 58, 292 (2007)

[22] Yu.T. Oganessian, V.K. Utyonkov, Nucl. Phys. A944, $62(2015)$

[23] M. Block, Nuclear Physics A 944, 471 (2015)
[24] H. Backe, W. Lauth, M. Block, M. Laatiaoui, Nuclear Physics A 944, 492 (2015)

[25] M. Asai, F.P. Heßberger, A. Lopez-Martens, Nucl. Phys. A944, 308 (2015)

[26] R.D. Herzberg, P. Greenlees, P. Butler, G. Jones, M. Venhart, I. Darby, S. Eeckhaudt, K. Eskola, T. Grahn, C. Gray-Jones et al., Nature 442, 896 (2006)

[27] S. Tandel, T. Khoo, D. Seweryniak, G. Mukherjee, I. Ahmad, B. Back, R. Blinstrup, M. Carpenter, J. Chapman, P. Chowdhury et al., Phys. Rev. Lett. 97, 082502 (2006)

[28] H. Jeppesen, R. Clark, K. Gregorich, A. Afanasjev, M. Ali, J. Allmond, C. Beausang, M. Cromaz, M. Deleplanque, I. Dragojević et al., Physical Review C 80, 034324 (2009)

[29] R. Clark, K. Gregorich, J. Berryman, M. Ali, J. Allmond, C. Beausang, M. Cromaz, M. Deleplanque, I. Dragojević, J. Dvorak et al., Physics Letters B 690, 19 (2010)

[30] F. Heßberger, S. Antalic, B. Sulignano, D. Ackermann, S. Heinz, S. Hofmann, B. Kindler, J. Khuyagbaatar, I. Kojouharov, P. Kuusiniemi et al., Eur. Phys. J. A 43, 55 (2010)

[31] A. Borschevsky, E. Eliav, M.J. Vilkas, Y. Ishikawa, U. Kaldor, Phys. Rev. A 75, 042514 (2007)

[32] V.A. Dzuba, M.S. Safronova, U.I. Safronova, Phys. Rev. A90, 012504 (2014)

[33] M. Laatiaoui et al., Nature 538, 495 (2016)

[34] P. Chhetri et al., Eur. Phys. J. D (2017 (under review))

[35] R. Ferrer, A. Barzakh, B. Bastin, R. Beerwerth, M. Block, P. Creemers, H. Grawe, R. de Groote, P. Delahaye, X. Fléchard et al., Nature Communications 8, 14520 (2017)

[36] S. Dmitriev, M. Itkis, Y. Oganessian, Status and perspectives of the Dubna superheavy element factory, in EPJ Web of Conferences (EDP Sciences, 2016), Vol. 131, p. 08001

[37] F. Déchery, A. Drouart, H. Savajols, J. Nolen, M. Authier, A. Amthor, D. Boutin, O. Delferriére, B. Gall, A. Hue et al., The European Physical Journal A 51, 66 (2015)

[38] W. Barth, K. Aulenbacher, M. Basten, F. Dziuba, V. Gettmann, M. Miski-Oglu, H. Podlech, S. Yaramyshev, A superconducting CW-LINAC for heavy ion acceleration at GSI, in EPJ Web of Conferences (EDP Sciences, 2017), Vol. 138, p. 01026

[39] T. Sato, M. Asai, A. Borschevsky, T. Stora, N. Sato, Y. Kaneya, K. Tsukada, C.E. Düllmann, K. Eberhardt, E. Eliav et al., Nature 520, 209 (2015) 